\title{
Las competencias en la Orientación del Siglo XXI: un acercamiento a la realidad costarricense
}

\section{Skills in the XXI Century Guidance: An Approach to the Costa Rican Reality}

\author{
Alejandra Gamboa Jiménez ${ }^{1}$ \\ División de Educación para el Trabajo, Centro de Investigación y Docencia en Educación (CIDE) \\ Universidad Nacional \\ Heredia, Costa Rica \\ lgamboa@una.ac.cr \\ Juan Ortega Rojas ${ }^{2}$ \\ Universidad Nacional \\ Heredia, Costa Rica \\ jortega1@costarricense.cr \\ Virginia Cerdas Montano \\ División de Educación para el Trabajo Centro de Investigación y Docencia en Educación \\ Universidad Nacional \\ Heredia, Costa Rica \\ vickycerdas@hotmail.com \\ Manuel Arturo Fallas Vargas ${ }^{4}$ \\ Académico de la Universidad Nacional \\ Heredia, Costa Rica \\ gutfallas@hotmail.com \\ Juan Vargas Fonseca ${ }^{5}$ \\ Ministerio de Educación Pública \\ San José, Costa Rica \\ juanvargas74@hotmail.com
}

Recibido 23 de agosto de 2010 • Aceptado 31 de agosto de 2010

\footnotetext{
Magíster en Psicopedagogía y Licenciada en Ciencias de la Educación con énfasis en Orientación. Orientadora del Ministerio de Educación Pública, Costa Rica, de primaria y secundaria. Académica de la Universidad Estatal a Distancia, Costa Rica. Académica, investigadora y extensionista de la Universidad Nacional, Costa Rica. Es subdirectora de la División de Educación para el Trabajo del Centro de Investigación y Docencia en Educación de la Universidad Nacional, Costa Rica.

2 Magíster en Psicología Clínica Grupal, Licenciado en Ciencias de la Educación con énfasis en Orientación. Orientador del Ministerio de Educación Pública, Costa Rica. Asesor Regional de Orientación de este mismo Ministerio. Actualmente académico de la Universidad Nacional, Costa Rica y orientador de la Universidad Estatal a Distancia, Costa Rica.

3 Magíster en Gestión y Liderazgo. Licenciada en Educación Ambiental y Administración Educativa. Docente en I y II ciclo del Ministerio de Educación Pública, Costa Rica. Directora de Escuela Primaria. Actualmente Directora de la División de Educación para el Trabajo del Centro de Investigación y Docencia en Educación (CIDE) de la Universidad Nacional, Costa Rica y académica de la Universidad Estatal a Distancia, Costa Rica.

4 Doctor en Sexualidad y Relaciones Interpersonales, Magíster en Psicopedagogía y Licenciado en Ciencias de la Educación con énfasis en Orientación. Orientador del Ministerio de Educación Pública, Costa Rica. Actualmente académico de la Universidad Nacional, Costa Rica.

5 Licenciado en Ciencias de la Educación con énfasis en Orientación. Licenciado en Administración Educativa y licenciado en Filosofía y Letras. Actualmente labora como orientador en el Ministerio de Educación Pública y como académico de la Universidad Nacional, Costa Rica.
} 
Resumen. El término de competencias, se ha venido utilizando desde hace algún tiempo en diversos campos, entre ellos y uno de los más importantes, el sistema educativo, desde los niveles preescolares y hasta la formación universitaria. Se entiende como un comportamiento integral de la persona que lo faculta para ingresar al mundo laboral de una forma más competitiva.

En el nivel universitario, se ha tratado de incluir el modelo por competencias como el eje transversal del proceso de enseñanza aprendizaje. En Europa, se ha implementado en la mayoría de sus universidades y en Latinoamérica, un número considerable de países, participación de este.

Se ha discutido ampliamente sobre la importancia de detectar tempranamente las competencias que poseen las poblaciones meta en educación; pero, en general, no se han considerado aquellas competencias que se pueden llamar "románticas o subjetivas" y que, desde la complejidad y competitividad actual, pareciera que carecen de valor.

Las competencias se han clasificado como genéricas o comunes y específicas, las cuales son propias de una rama del saber. En Orientación, específicamente, se han determinado aquellas competencias generales y específicas deseables para un buen desempeño laboral.

El artículo contempla los principales antecedentes de las competencias y analiza este enfoque desde la disciplina de la Orientación. Sobre este punto, se realizó una investigación con profesionales en Orientación, la cual concluye con algunas de las competencias que se consideran indispensables para ejercer esta disciplina. No obstante, las competencias del estudio apuntan, mayoritariamente, a competencias genéricas, más que específicas.

Palabras clave. Competencias, competencias en Orientación, orientación educativa y vocacional, competencias genéricas y específicas, educación costarricense, diversidad.

Abstract. During a long time, the term competence has been used in different fields. Among those fields, and one of the most important, we can mention the educational system, from preschool to university levels. The term has to be understood as one person integral behavior, making him/her able to enter the labor force in the most competitive way.

Within the university realm, experts have tried to include the model of competences as a transversal axle into the teaching-learning process. In European universities, such model has been implemented in most of them. In Latin America universities, on the other hand, it has been used in a good number of countries.

The importance of early detection of competences among target populations in education has been widely discussed. However, it has been highlighted the necessity of considering romantic or subjective competences, which seem to lack of value in this complex and competitive actual world.

The term competence has been classified as genetic competence, or common and specific competence. The latter is related to the knowledge scope. Within the counseling field, specifically, the general and specific competences are deemed highly appropriate for good a work performance.

This article focuses on the main antecedents of competences and analyzes this approach from counseling field perspective. In regards to this specific aspect, a survey with professionals in counseling was carried out. Such a survey ends up dealing with some of the competences considered unavoidable for an accurate performance of professionals in counseling. It is important to mention that the competences of the survey mainly point out to genetic competences rather than to specific competences.

Key word. Competences, educational and vocational counseling, genetic and specific competences, Costa Rican education, diversity.

\section{Introducción}

Lo que se conoce hasta hace poco como un modelo de competencias en la educación superior es realmente, en la práctica, un enfoque, debido a que se basa en el desempeño de los estudiantes, trabajadores y profesionales en servicio. Por otro lado, el modelo pedagógico toma en cuenta todo 
el proceso de gestión curricular, la didáctica, la enseñanza y la evaluación (Tobón, 2010) y todavía, en nuestro país, se encuentra en proceso de este alcance.

El actual enfoque por competencias tiene sus orígenes en la sociedad desde los griegos. En el siglo XX comenzaron a presentarse en la academia universitaria con varios proyectos en Alemania y Estados Unidos (Tobón, 2010). En el contexto europeo, sobre la base de la Declaración de Bolonia, (Declaración Conjunta de los Ministros Europeos de Educación, 1999), se convirtió en un proceso de discusión, confluencia y análisis de la educación superior europea. Posteriormente, se encuentra la referencia al Tuning Europeo, (González, y Wagenaar, 2008) que es la participación de 175 universidades europeas en favor de dar respuesta a los desafíos planteados en la Declaración mencionada. Se basó en las necesidades de la sociedad, en la actualidad, y en prospectiva, a los retos que se visualizan para el futuro.

Asimismo, la Declaración de Bolonia, (1999) referida como el "Proceso Bolonia", (González, J. y Wagenaar, 2008) tiene unos antecedentes que se pueden visualizar en el Tabla 1, donde se reflejan las diferentes declaraciones y comunicados de los ministros o de sus representaciones oficiales, autoridades de educación superior europeas. Las mismas datan del año 1998 en la Sorbona (Ministros representantes de Francia, Alemania, Italia y Reino Unido. (1998), en Bolonia, (Ministros Europeos de Educación, 1999), en Praga (Ministros Europeos en Funciones de la Educación Superior, 2001), en Berlín, (Ministros de Educación Superior, 2003), en Londres (Ministros de Educación Superior, 2007) y, finalmenten en Lovaina (Ministros Europeos de Educación Superior, 2009).

Como parte de este proceso, se da una iniciativa en América Latina, conocida como el TuningAmérica Latina, (Benitone, P., Esquetini, C., González, J., Maletá, M. M., Siufi, G., Wagenaar, R. (2007), quien tuvo la cooperación de 19 países en el proceso y a la vez presenta desafíos semejantes al Tuning-Europa que se pueden resumir en:

- Calidad de la educación superior.

- Acuerdos, convergencia, pertinencia, comparabilidad, competitividad y transparencia en la educación superior.

- Cooperación internacional basada en la movilidad estudiantil y del profesorado.

- Homologación de titulaciones, no solo en contenidos y conocimientos, sino también en competencias generales y específicas.

La definición de Tuning sobre competencias refiere al concepto inglés “tune”, el cual significa sintonizar una frecuencia determinada en la radio; pero también se utiliza para describir la "afinación" de los distintos instrumentos de una orquesta, de modo que los intérpretes puedan interpretar la música sin disonancias (González y Wagenaar (2008).

En relación con este elemento sobre las competencias, se pretende profundizar en este estudio. Según el Tuning para América Latina, existe una serie de competencias que se pueden desarrollar mediante la formación universitaria y que son consecuentes con los avances y demandas de la sociedad actual. El estudio que da origen a este artículo se centra, con especial interés, en las competencias que se requieren en el profesional de la Orientación en el contexto costarricense y se realiza contextualmente, debido a que la Orientación en este país posee un campo laboral diversificado.

Específicamente, la orientación escolar tiene 46 años de existencia. se inicia en el sistema educativo público y trasciende a escenarios laborales típicos tales como la educación primaria y secundaria, educación superior, colegios universitarios, Instituto Nacional de Aprendizaje, Instituto Mixto de Ayuda Social, Ministerio de Justicia, Hogares de Adultos Mayores y organizaciones gubernamentales y no gubernamentales. 
Con la expansión del campo laboral de la Orientación y teniendo como pilar la orientación vocacional como el espacio especializado y vital de esta disciplina, además de la prevención como campo delimitado en el accionar del profesional o la profesional de la Orientación y sobre la base del trabajo en desarrollo humano durante todas las etapas del ciclo vital en el cual se desempeña, se identifican una serie de competencias deseables que debe poseer dicho profesional y que tratarán de ampliarse a lo largo de este estudio.

Tabla 1

Resumen de Declaraciones y Comunicados Proceso Bolonia. Espacio Europeo de Educación Superior

\begin{tabular}{|c|c|l|}
\hline Año & Declaración & \multicolumn{1}{c|}{ Síntesis Declarativa } \\
\hline 1998 & Declaración de Sorbona & $\begin{array}{l}\text { Diseño del Sistema de Educación Superior. Visualización de cambios para } \\
\text { las condiciones educativas y laborales. Invitación a otros países para unirse } \\
\text { a los retos planteados en la Declaración. }\end{array}$ \\
\hline 1999 & Declaración de Bolonia & $\begin{array}{l}\text { Fomentar la movilidad y empleabilidad del ciudadano y el desarrollo glo- } \\
\text { bal del continente europeo, la enseñanza superior y la investigación deben } \\
\text { adaptarse a las expectativas de la sociedad y a los nuevos conocimientos } \\
\text { científicos. }\end{array}$ \\
\hline 2001 & Declaración de Praga & $\begin{array}{l}\text { Establecimiento de estrategias en la educación superior para garantizar la } \\
\text { calidad que mejore la compatibilidad entre países y la competitividad de la } \\
\text { educación superior europea. }\end{array}$ \\
\hline 2005 & Declaración de Bergen & $\begin{array}{l}\text { Las necesidades de incrementar la competitividad debe conciliarse con } \\
\text { la necesidad de mejorar las características sociales del área de Educación } \\
\text { Superior Europea. Se pretende que exista una cohesión social que reduzca } \\
\text { las desigualdades sociales y de género. }\end{array}$ \\
\hline 2007 & Declaración de Londres & $\begin{array}{l}\text { Movilidad del profesorado y el estudiantado en el mundo, garantizando } \\
\text { acreditación y garantía de calidad. Se recalca la importancia de la inves- } \\
\text { tigación universitaria y el apoyo de la educación superior al desarrollo } \\
\text { económico, cultural, y social de los países. }\end{array}$ \\
\hline $\begin{array}{l}\text { Materialización del Espacio Europeo de Educación Superior (EEES), } \\
\text { caracterizado por la autonomía universitaria, la libertad académica, la } \\
\text { libertad de oportunidades y los principios democráticos, que facilitarán } \\
\text { movilidad, empleabilidad, competitividad. El EEES debe adaptarse perma- } \\
\text { nentemente para garantizar competitividad y responder eficazmente a los } \\
\text { retos de la globalización. }\end{array}$ \\
\hline Declaración de Lovaina \\
$\begin{array}{l}\text { Proceso hacia el año 2020, donde se vinculan los esfuerzos los nuevos desa- } \\
\text { fíos de enfrentar el desarrollo acelerado de la tecnología. Los principales } \\
\text { acuerdos son en torno a la innovación, empleabilidad, y movilidad. }\end{array}$ \\
\hline
\end{tabular}

Nota. Elaboración propia a partir de las declaraciones y comunicados de las representaciones oficiales de las autoridades de educación superior en el espacio de educación superior europeo.

Existe en la bibliografía, variadas formas de conceptualizar y categorizar las competencias. Cada conceptualización estudiada y analizada, concluyen los autores de este estudio, refiere a 
cierta intencionalidad o funcionalidad de quien la ha creado; por ello, se decide por una propia conceptualización, basada en los cuatro saberes expuestos por Delors, 1996.

Para contextualizar, Delors, quien fue ex ministro de Francia, investigador y comisionado de la UNESCO, en el año 1996, con su alta experiencia en educación empoderó el informe La educación encierra un tesoro, (Delors, 1996) más conocido por los cuatro pilares de la educación a lo largo de la vida. Hace un llamado a la necesidad de una educación centrada en el estudiantado como protagonista de su propia historia, preparado para vivir y enfrentar la vida con herramientas que va obteniendo y potencializando a través de su ciclo vital. Este hermoso informe trasciende en la labor orientadora que desempeñan cientos de profesionales costarricenses de esta disciplina, quienes intentan ir más allá del sistema riguroso y utópicamente flexible que se desarrolla en nuestro contexto. Los cuatro saberes que establece Delors (1996) son: aprender a ser, aprender a hacer, aprender a conocer y aprender a convivir. Desde el enfoque por competencias en que se fundamenta este estudio, se les puede comprender como las competencias deseables para el profesional o la profesional en Orientación del Siglo XXI, pues al fin de cuentas se resumen en "el saber vivir".

Saber ser desde el conocimiento del propio yo, conociendo y reconociendo las propias fortalezas y debilidades, sentir, pensar y transformarse continuamente.

Saber hacer con responsabilidad y ética, adaptándose a la complejidad del contexto dinámico y complejo.

Saber conocer lo que existe en materia de la disciplina, e innovar y aportar desde la propia práctica.

Saber convivir en un mundo diverso, inclusivo, donde todas las personas tienen un lugar.

Por ello, en Orientación se entenderá como competencia: aquella capacidad para responder a un contexto complejo y dinámico, donde la persona profesional es conocedora de su campo de acción, principalmente en el área vocacional, y su práctica se encuentra impregnada por la ética, la responsabilidad y se sustenta en principios humanistas y científicos.

\section{Competencias: Un panorama general}

\section{Origen del concepto}

En la actualidad, el enfoque de las competencias es bastante común entre quienes laboran directa o indirectamente en el ámbito educativo. Para algunos es un concepto nuevo y para otros es un nombre que se le da a algo que ya se conocía y se había venido trabajando desde hace mucho tiempo.

Anteriormente a la divulgación y conceptualización del enfoque por competencias, se le ha conocido como habilidades, valores e intereses, creencias y actitudes, motivaciones, aptitudes y rasgos de personalidad; conceptos que, a partir del modelo por competencias en estudio, se constituyen en parte de la conceptualización como elementos de las competencias.

Diferentes teóricos coinciden al definir competencia como aquellas habilidades integrales en que se logran incorporar el saber ser (ética, valores), el saber hacer (habilidades, destrezas) y el saber conocer (habilidades cognitivas).

Ruiz (2002) aporta una serie de definiciones de competencias, dentro de las cuales la primera de ellas las define como "(...) las capacidades con que un sujeto cuenta para ejercer determinados desempeños" (p. 87). 
¿De dónde viene este nuevo término? De acuerdo con Tobón (2006) este término surge:

En la emergencia del concepto de competencias ha influido la traslación del sentido de competitividad del mundo empresarial al ámbito de la educación, con la filosofía de formar personas competentes para responder a los requerimientos del mercado laboral, el cual contribuye a posicionar y validar este concepto (...) en dicha traslación se encuentra de fondo un manejo del poder de la economía, en tanto que el sector empresarial posibilita el empleo y favorece el desarrollo del país. En este sentido el lenguaje empresarial es legitimado en el discurso social". (p. 91)

Pareciera, entonces, que los sistemas educativos responden a una estructura externa que le dicta la coyuntura sobre las líneas a seguir según sus necesidades. Por ello, todo el enfoque por competencias se encuentra en respuesta a los sistemas económicos, políticos y sociales de los países. Girox (1999), citado por Tobón, 2006, plantea que el sistema educativo está fuertemente unido al contexto socioeconómico en el que se sitúa. En este sentido se podría señalar que el discurso educativo responde a las estructuras sociales y políticas del momento histórico.

Desde esta perspectiva, conviene tratar de definir el concepto de competencias desde lo que se ha llamado competencias genéricas y competencias específicas:

En relación a las primeras, la Universidad Católica de Temuco en Chile (2005), en su proyecto de incorporación del modelo de competencias a su proceso de enseñanza las define como:

(...) aquellas que permiten el desarrollo de las personas, tanto en su dimensión intrapersonal como de interacción con otros. Estas competencias se encuentran presentes en la realización de numerosas y variadas actividades y en diferentes contextos, y debieran ser ejes transversales para todo currículo. (p. 9)

La anterior definición bien se podría adaptar en cualquier nivel de la educación, desde el sistema preescolar al universitario. En el caso específico de este último, dentro del proyecto citado, se menciona que las competencias genéricas "(...) identifican los elementos comunes que pueden ser compartidos con cualquier carrera" (Universidad de Temuco, 2005, p. 9). Asimismo, las competencias específicas son aquellas que reflejan el desempeño propio de cada disciplina. Tal definición, en los niveles de educación general básica y diversificada, se aplica a las diferentes asignaturas de los planes curriculares.

\section{Detección temprana y desarrollo de competencias}

A partir de la necesidad de que, una vez concluidos los estudios secundarios los jóvenes y las jóvenes hagan elecciones vocacionales acordes con su perfil de competencias, se requiere implementar estrategias que faciliten la detección temprana de sus competencias.

Ruiz (2002) sugiere guías de trabajo que faciliten tal exploración y que contemplen trabajos individuales, actividades libres y que requieran ejercitar diversas clases de razonamiento, resolución de problemas, discusiones y debates, trabajo en pequeños grupos, trabajos escritos y orales, dramatizaciones, entre otras.

En el caso particular de la disciplina de Orientación, cuya base teórica se centra en el desarrollo vocacional, quedaría a creatividad del profesional la creación y aplicación de técnicas que promuevan la autoexploración de competencias, así como el desarrollo de las mismas. 
Sobre el punto anterior y desde las diversas manifestaciones de las competencias en el ser, conocer, hacer y convivir, se pretende que las personas desarrollen las propias, en procura de encontrar un lugar dentro de un medio que cada vez resulta más exigente.

Por lo general la exigencia de tener claridad en el para qué se es competente, va dirigida a la elección de un oficio u profesión. El desconocimiento de sí mismo implicaría un gran obstáculo en la incorporación al medio, no sólo en lo laboral, sino también en la creación de vínculos, si hablamos desde el ámbito del ser.

Al respecto Ruiz (2002) plantea que al finalizar el último año escolar el joven y la joven se enfrentan a la decisión de escoger una ocupación acorde con sus capacidades, preferencias y destrezas. Apunta la autora que la poca o mucha claridad de estos elementos repercute en el ver logrados o truncados los sueños.

Desde esta perspectiva, se visualiza la importancia de detectar y desarrollar competencias desde edades tempranas, que lleven a la persona a hacer elecciones acordes con su perfil y con el sentido que se le quiera dar a la existencia.

Las principales competencias genéricas que se conciben, en primer lugar, son las relacionadas con el uso de la tecnología, el manejo de la información, los idiomas y, con mayor importancia, relaciones interpersonales basadas en el respeto a la diversidad.

Vale resaltar la necesidad de que quienes faciliten la detección y desarrollo de competencias estén libres del prejuicio en relación con escalas de valoración de competencias predeterminadas; y darle más importancia a los ámbitos del ser, conocer, hacer y convivir.

Abdón (2003) aporta:

(...) si se desea una educación por competencias como enfoque alternativo, el concepto de competencias es más amplio: ser competente es saber hacer y saber actuar entendiendo lo que se hace, comprendiendo cómo se actúa, asumiendo de manera responsable las implicaciones y consecuencias de las acciones realizadas y transformando los contextos a favor del bienestar humano. (p. 12)

\section{Competencias y diversidad}

Hablar de competencias nos lleva casi de inmediato a pensar en competir o competir con otros. Y sabemos que como resultado de competir aparece la persona ganadora y la perdedora. Visto de esta forma, pareciera que las llamadas discapacidades vendrían a colocar a quien es poseedor de la misma en el segundo puesto, en el que nadie quiere estar, el del perdedor.

De nuevo surge la pregunta: ¿para qué se educa en competencias?, ¿para un mundo competitivo, donde el saber conocer y el saber hacer predomina; o bien, donde el saber ser y el saber convivir tienen un espacio preponderante en un mundo rico en diversidad?

Sobre lo anterior, Ruiz (2002) plantea que:

(...) es importante aclarar que tanto genios como autistas, personas con síndrome de Down, estrábicos, disléxicos o hiperactivos, requieren que los educadores posean preparación especializada o capacitación adecuada, tanto para detectar la existencia de tales limitaciones como para trabajar con ello... pues toda persona con deseo y posibilidad de aprender debe tener acceso a una oportunidad para demostrar sus capacidades. (p. 25) 
Sobre esta misma línea, la autora anota que las personas con autismo poseen grandes habilidades cerebrales y de pensamiento, además de una memoria prodigiosa; que los mejores taxistas u operarios para máquinas y herramientas son los que presentan síndrome de Down; que las personas que presentan déficit atencional resultan ideales para desactivar artefactos explosivos, debido a que su ritmo cardíaco es más lento que el común, lo cual hace que no se inquieten por nada, lo que a su vez produce que sus manos no tiemblen en situaciones extremas.

Con las ideas anteriores, se concluye que ciertos rasgos vistos como grandes limitaciones, desde otro lugar podrían mirarse como fortalezas al servicio propio y de los otros, El enfoque de competencias resulta discriminativo solo si se ubica en un mundo que rechaza a quien no cumple con lo estipulado dentro de la norma.

Dentro de los sistemas económicos y políticos de una sociedad basada en competencias, donde la generación del conocimiento y, por ende, una respuesta a la productividad y el consumismo, pareciera no tener asidero la idea de la inclusión y la diversidad ni mucho menos la discapacidad. Desde este nuevo paradigma, es la sociedad la que presenta la discapacidad y no la persona con alguna de ellas, así que existe el nuevo reto de enfrentar el mundo basado en competencias. No obstante, desde los principios de este estudio sí es posible tener un lugar en la sociedad y no necesariamente el del excluido.

\section{Competencias básicas e implicaciones educativas de un modelo por competencias}

Asumir un enfoque educativo por competencias implica tener claridad no solo de las competencias que se pretenden desarrollar, sino también de las condiciones que se requieren para su desarrollo.

Solo sobre el primer punto, Abdón (2003) considera 4 dimensiones dentro de las cuales ubica algunas de las competencias básicas.

\section{Dimensión biológica}

- Desplazarse en forma coordinada a través del espacio, percibiendo los estímulos del ambiente y conservando el sentido de ubicación.

\section{Dimensión intelectual}

- Comunicarse en lenguaje natural y en cualquier otra forma de representaciones simbólicas

- Producir inferencias válidas a partir de premisas, mediante el uso de sistemas de razonamiento.

- Elaborar conceptos a través de relaciones empírico-teóricas.

- Diseñar, transferir y utilizar tecnología para mejorar sus condiciones de vida.

\section{Dimensión social}

- Interactuar de manera armónica con otras personas, conservando la autonomía, practicando la cooperación y desarrollando lazos de afecto y solidaridad.

- Valorar de manera equilibrada las actuaciones propias y las ajenas.

- Apreciar la armonía y la coherencia como fundamento de la belleza de las cosas, las personas, sus actuaciones y sus obras. 


\section{Dimensión interpersonal}

- Conocerse a sí mismo, identificar las partes del cuerpo, el estado de sus funciones orgánicas, el nivel de bienestar o malestar que pueda sentir en determinado momento, la forma como reacciona ante las situaciones, sus emociones, sus sentimientos y la conciencia y control de su proceso cognitivo.

En relación con las dimensiones anteriores con sus respectivas competencias, el mismo autor considera que la institución que decida trabajar con un enfoque por competencias debe tener una serie de condiciones relacionadas con espacios amplios y con recursos para practicar deportes, para el arte, para la ciencia, para la tecnología y para la interacción social.

En relación con el recurso humano anota algo sumamente importante: que las personas encargadas de desarrollar este enfoque tienen, obligatoriamente, que ser poseedoras de las competencias genéricas planteadas antes, así como las competencias específicas que les permitan un desempeño eficiente de acuerdo con el enfoque adoptado.

Sobre la base de las competencias anteriormente expuestas, resulta de primordial importancia practicar y manifestar una congruencia entre lo que se es y lo que se pretende enseñar o desarrollar.

En este sentido, Abdón (2003) plantea que asumir un enfoque por competencias requiere "(...) un sistema educativo vigoroso, en donde lo mejor de los recursos de la sociedad incluido el talento humano, estén destinados de manera prioritaria a la tarea de educar a las nuevas generaciones" (p. 30).

Resulta, entonces, muy pertinente conocer la realidad tanto personal como institucional, si se pretende trabajar con este enfoque. En otras palabras, es necesario conocer con qué recursos personales y externos se cuenta antes de plantearse expectativas que en la propia realidad podrían ser inalcanzables.

\section{Las competencias del ser: lo romántico del existir}

\section{¿Te parece romántico ver las estrellas?}

Relacionar la pregunta anterior con la temática del presente artículo, quizá no resulte fácil. Se podría preguntar: ¿Y qué tiene que ver el que a alguien le parezca romántico o no ver las estrellas, con el tema de competencias?

Con los antecedentes y las premisas presentadas hasta ahora, es probable que el lector o lectora ubique el tema y visualice una serie de competencias genéricas o específicas que resulten imprescindibles de desarrollar en la propia persona y en los otros, con fines competitivos y en función de un mercado que involucra, de forma integral, al ser humano y que le exige ser competente en las mencionadas dimensiones y saberes de la competencias.

Este ajetreo lleva, en muchos casos, al olvido de desarrollar esas competencias llamadas "románticas" y que podrían ubicarse en cualquiera de las áreas mencionadas, especialmente, en las del saber ser y saber convivir. Quizá estas competencias, en primera instancia, no resulten indispensables para conseguir un buen trabajo, pero en el trascurso de la vida, sí podrían contribuir a crear mejores vínculos sociales y afectivos con la propia persona y con las otras.

Se refiere a esas experiencias tan gratificantes para quienes han aprendido a valorarlas: darse el tiempo para mirar las estrellas, ver un atardecer, apreciar el aroma de la flores, caminar descalzo por la hierba, entre otras muchas experiencias que en soledad alimentan el espíritu y, por ende, el ser; y que, en compañía de otra persona, fortalecen los vínculos afectivos, tan importantes en ese 
lapso de tiempo llamado vida que a veces se olvida por vivir en función de los mandatos sociales que dictan los "deber ser". Estas competencias también tienen fundamentos teóricos que refieren a lo que Gardner (1998) como inteligencias intrapersonal, interpersonal y naturalista. Ubican a las personas con estas inteligencias más desarrolladas, en profesiones, oficios y empleos con ventajas de desarrollo óptimo. Estas personas despliegan relaciones interpersonales, producto de un desarrollado conocimiento de sí mismas, interpretado como intrapersonal y con acercamientos naturalistas y metafísicos o espirituales.

\section{Competencias genéricas y específicas para profesionales en Orientación}

La Asociación Internacional para la Orientación Educativa y Profesional (AIOEP), en el año 1999 comenzó el desarrollo de una serie de competencias internacionales que los profesionales necesitan para proporcionar servicios de orientación educativa y profesional, con garantía de un servicio profesional de calidad. La serie de competencias fue validada por una bolsa de 700 profesionales que trabajan en diferentes empleos, de 38 países. Dichas competencias fueron adoptadas por la Asamblea General en septiembre de 2003. El Profesional de la Orientación Educativa y Profesional (AIOEP) nació de estas competencias para identificar a aquellos individuos con una educación y experiencia documentada en la orientación educativa y profesional (AIOEP, 2008), cuyo objetivo es lograr una credencial internacional en la práctica de orientación educativa y profesional.

Esta asociación conceptualiza lo que se conoce como competencias genéricas con el nombre de competencias centrales, estas implican los conocimientos, habilidades y actitudes. Además, retoma las competencias conocidas como específicas con el nombre de competencias especializadas, las cuales se encuentran en función de tareas que se desempeñan (ver Tabla 2). Asimismo, esta asociación utiliza el concepto de cliente, el cual se tomará literalmente del texto; no obstante, los autores entienden como cliente a la persona quien recibe el servicio profesional de Orientación.

\section{Competencias centrales (genéricas)}

- Demostrar profesionalidad y comportamiento ético en el desempeño de sus tareas.

- Promover en los clientes el aprendizaje y el desarrollo personal y de la carrera.

- Apreciar y atender las diferencias culturales de los clientes, posibilitando la interacción efectiva con poblaciones diversas.

- Integrar la teoría y la investigación en la práctica de la orientación.

- Poseer habilidad para diseñar, implementar y evaluar intervenciones y programas de orientación.

- Ser consciente de las propias capacidades y limitaciones.

- Mostrar habilidad para usar el nivel apropiado de lenguaje para comunicarse con colegas o clientes, logrando así una comunicación eficaz.

- Tener conocimiento de información actualizada sobre educación, formación, tendencias de empleo, mercado de trabajo y asuntos sociales.

- Demostrar sensibilidad social e inter-cultural.

- Mostrar habilidad para cooperar de manera eficaz con un grupo de profesionales.

- Demostrar conocimiento sobre el desarrollo evolutivo de la persona. 
Tabla 2

Competencias especializadas (específicas). Adaptado con fines esquemáticos de AIOEP, 2008

\begin{tabular}{|c|c|c|}
\hline 1. Diagnóstico & 2. Orientación Educativa & 3. Desarrollo de la carrera \\
\hline $\begin{array}{l}\text {-Definir y diagnosticar con } \\
\text { rigurosidad y acierto las } \\
\text { necesidades de los clientes } \\
\text { basándose en diferentes } \\
\text { instrumentos y técnicas de } \\
\text { diagnóstico. } \\
\text {-Usar los datos e información reco- } \\
\text { gidos en el diagnóstico de manera } \\
\text { apropiada de acuerdo con la situa- } \\
\text { ción. } \\
\text {-Identificar las situaciones que } \\
\text { requieran ser derivados a servicios } \\
\text { especiales de apoyo. } \\
\text {-Facilitar el contacto entre los } \\
\text { clientes y los servicios especiales } \\
\text { de apoyo. a los que se remite. } \\
\text {-Mantener información actualizada } \\
\text { sobre los servicios de apoyo. } \\
\text {-Llevar a cabo un análisis del } \\
\text { contexto en el que está inmerso el } \\
\text { cliente. }\end{array}$ & $\begin{array}{l}\text {-Demostrar compromiso con las } \\
\text { posibilidades y habilidades de } \\
\text { los estudiantes para facilitar su } \\
\text { desarrollo. } \\
\text {-Guiar a los individuos y grupos } \\
\text { de estudiantes en el desarrollo de } \\
\text { sus planes educativos. } \\
\text {-Ayudar a los estudiantes en el } \\
\text { proceso de toma de decisiones. } \\
\text {-Ayudar a los estudiantes a } \\
\text { conocerse mejor. } \\
\text {-Asesorar a los estudiantes } \\
\text { en la selección de itinerarios } \\
\text { académicos. } \\
\text {-Ayudar a los estudiantes a } \\
\text { superar las dificultades de } \\
\text { aprendizaje. } \\
\text {-Motivar y ayudar a los } \\
\text { estudiantes a participar en } \\
\text { intercambios internacionales. } \\
\text {-Consultar a los padres sobre } \\
\text { el desarrollo y los progresos } \\
\text { educativos de sus hijos. } \\
\text {-Ayudar a los estudiantes a } \\
\text { mejorar las metodologías de } \\
\text { enseñanza. } \\
\text {-Ayudar a los profesores a } \\
\text { implementar la orientación en el } \\
\text { currículum. }\end{array}$ & 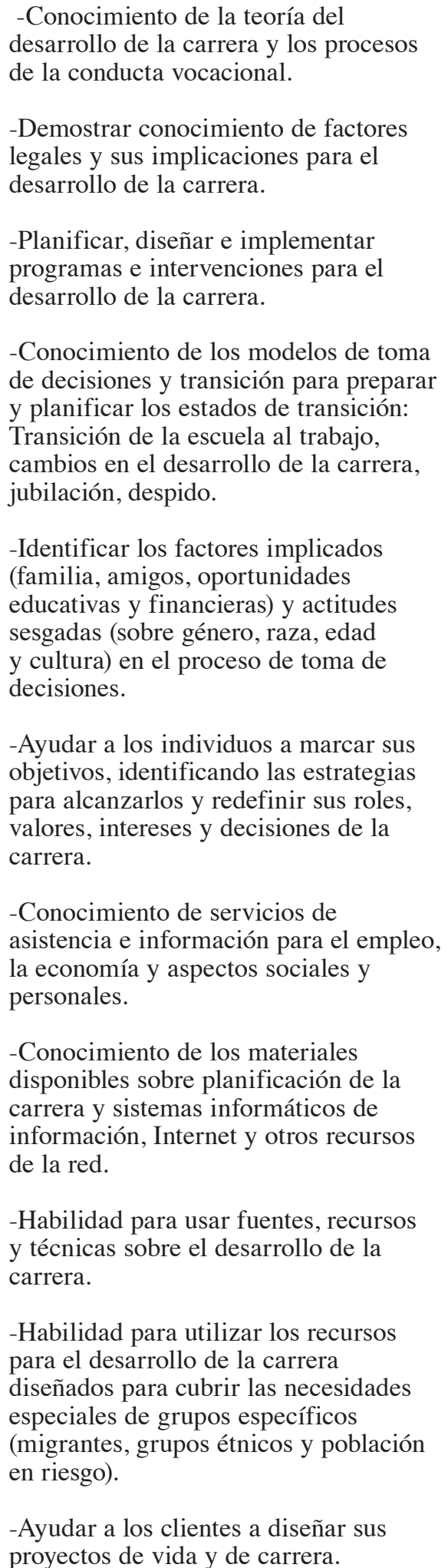 \\
\hline
\end{tabular}




\begin{tabular}{|c|c|c|c|}
\hline & \multicolumn{2}{|l|}{ 5. Información } & \\
\hline $\begin{array}{l}\text {-Uso de técnicas grupales de orien- } \\
\text { tación. } \\
\text {-Cubrir las necesidades de los } \\
\text { estudiantes en riesgo. } \\
\text {-Ayudar a los clientes en: } \\
\text { prevención de problemas persona- } \\
\text { les, desarrollo de la personalidad, } \\
\text { resolución de problemas, toma de } \\
\text { decisiones, identidad sexual, habi- } \\
\text { lidades sociales, educación para la } \\
\text { salud, uso del tiempo libre. } \\
\text {-Ayudar a los clientes a desarrollar } \\
\text { su plan de carrera. }\end{array}$ & $\begin{array}{l}\text {-Conocimiento de le } \\
\text { sobre educación, for } \\
\text { trabajo a nivel local, } \\
\text { internacional. } \\
\text {-Conocimiento de e } \\
\text { de títulos y cualifica } \\
\text { profesionales en dife } \\
\text {-Recopilar, organiza } \\
\text { información actualiz } \\
\text { desarrollo personal, } \\
\text { carrera, especialmer } \\
\text {-Educación y forma } \\
\text { información ocupac } \\
\text { oportunidades de en }\end{array}$ & $\begin{array}{l}\text { slación } \\
\text { ación y } \\
\text { acional e } \\
\text { ivalencia } \\
\text { ones } \\
\text { ntes países. } \\
\text { y distribuir } \\
\text { da sobre el } \\
\text { cial y de la } \\
\text { : } \\
\text { fn, } \\
\text { nal y } \\
\text { leo. }\end{array}$ & $\begin{array}{l}\text {-Consultar con los padres, profesores, } \\
\text { tutores, trabajadores sociales, } \\
\text { administradores y otros agentes para } \\
\text { "mejorar" su trabajo con los estudiantes. } \\
\text {-Demostrar habilidades interpersonales } \\
\text { para crear y mantener la relación de } \\
\text { consulta, alcanzar los objetivos y el } \\
\text { cambio de comportamiento. } \\
\text {-Demostrar habilidad para trabajar con } \\
\text { organizaciones (universidades, negocios, } \\
\text { municipios y otras instituciones). } \\
\text {-Interpretar y explicar conceptos y } \\
\text { nueva información de manera efectiva. } \\
\text {-Coordinar al personal y la comunidad } \\
\text { para "proporcionar" recursos a los } \\
\text { estudiantes. } \\
\text {-Asesorar a los estudiantes en el } \\
\text { acceso y uso de programas y servicios } \\
\text { especiales y de grupos de apoyo. } \\
\text {-Habilidad para coordinar y estimular } \\
\text { la creatividad del estudiante para } \\
\text { diseñar su propio programa (educativo y } \\
\text { vocacional). } \\
\text {-Habilidad para crear una buena imagen }\end{array}$ \\
\hline \multicolumn{2}{|l|}{ 7. Investigación } & \multicolumn{2}{|c|}{ 8. Gestión de Programas y Servicios } \\
\hline $\begin{array}{l}\text {-Evaluar los programas e intervenci } \\
\text { aplicando técnicas actualizadas y m } \\
\text { de programas. } \\
\text {-Mantener información actualizada } \\
\text { de las investigaciones. }\end{array}$ & $\begin{array}{l}\text { igación, recogida y } \\
\text { n sobre orientación. } \\
\text { ra informar los resul- } \\
\text { estigaciones. } \\
\text { igaciones en la prác- } \\
\text { ones de orientación, } \\
\text { odelos de evaluación } \\
\text { sobre los resultados }\end{array}$ & $\begin{array}{l}\text {-Identificar } \\
\text {-Conducir } \\
\text {-Inventaria } \\
\text { ción e impl } \\
\text {-Conocimi } \\
\text {-Promover } \\
\text { el servicio } \\
\text {-Uso (diseñ } \\
\text { gramas e ir } \\
\text {-Evaluar la }\end{array}$ & $\begin{array}{l}\text { las poblaciones objeto del estudio } \\
\text { análisis de necesidades } \\
\text { los recursos relevantes para la planifica- } \\
\text { mentación de programas } \\
\text { nto de bibliografía relevante y actualizada } \\
\text { l interés comunitario sobre el programa o } \\
\text {, implementación y supervisión) de pro- } \\
\text { ervenciones } \\
\text { efectividadod de las intervenciones } \\
\text { ilidad para organizar y gestionar servicios de } \\
\text { ducativa, personal, vocacional y de empleo } \\
\text { supervisar el personal de los servicios } \\
\text { l desarrollo del personal. }\end{array}$ \\
\hline
\end{tabular}




\begin{tabular}{|c|c|}
\hline 9. Desarrollo comunitario & 8. Gestión de Programas y Servicios \\
\hline $\begin{array}{l}\text {-Mostrar habilidad para establecer contacto con miem- } \\
\text { bros de la comunidad. }\end{array}$ & $\begin{array}{l}\text {-Asesorar al cliente en estrategias de búsqueda de } \\
\text { empleo. }\end{array}$ \\
\hline -Analizar los recursos humanos y materiales de la comu- & -Usar Internet en el proceso de búsqueda de empleo. \\
\hline $\begin{array}{l}\text {-Llevar a cabo un análisis de necesidades de la comuni- } \\
\text { dad. }\end{array}$ & $\begin{array}{l}\text {-Presentar a los clientes la oportunidades de empleo } \\
\text { disponibles para su perfil y facilitar la selección } \\
\text { apropiada. }\end{array}$ \\
\hline $\begin{array}{l}\text {-Trabajar con la comunidad para el uso efectivo de los } \\
\text { recursos de acuerdo con las necesidades. }\end{array}$ & $\begin{array}{l}\text {-Contactar con empleadores y centros formativos } \\
\text { para obtener información sobre sus servicios. }\end{array}$ \\
\hline $\begin{array}{l}\text {-Trabajar con la comunidad para desarrollar, implementar } \\
\text { y evaluar planes de acción con miras de mejora económi- } \\
\text { ca, social, educativa y del empleo. }\end{array}$ & $\begin{array}{l}\text {-Consultar a expertos en regulación y legislación. } \\
\text {-Dar seguimiento del cliente en el empleo. }\end{array}$ \\
\hline $\begin{array}{l}\text {-Cooperar con instituciones nacionales e internacionales } \\
\text { de orientación educativa y profesional. }\end{array}$ & $\begin{array}{l}\text {-Seleccionar los individuos apropiados para cubrir } \\
\text { determinadas plazas de empleo o formación } \\
\text {-Asesorar a los clientes en el mantenimiento del } \\
\text { empleo. }\end{array}$ \\
\hline
\end{tabular}

Nota. Elaboración esquematiza a partir de las competencias establecidas por la Asociación Internacional para la Orientación Educativa y Profesional (AIOEP, 2008).

En la Tabla 2, se especifican las competencias especializadas o específicas, categorizadas por dicha Asociación en competencias de diagnóstico, orientación educativa, desarrollo de la carrera, counseling, información, consulta, investigación, gestión de programas y servicios, desarrollo comunitario y empleo; las mismas se pueden adaptar al contexto costarricense.

Para realizar la contextualización al ámbito costarricense, se realizó una consulta a profesionales en servicio de la Orientación del sector educativo de la escuela primaria y secundaria, y a la Asesoría Nacional de Orientación, con el objetivo de conocer las competencias que se consideran deseables, tanto en el perfil del profesional en Orientación, como las que se consideran necesarias en la población meta de esta disciplina.

La consulta fue aplicada a cincuenta profesionales de la Orientación que se desempeñan en la Asesoría Nacional y Regional de Orientación en educación secundaria y primaria. El instrumento aplicado fue un cuestionario denominado "Percepción de competencias en la orientación", instrumento elaborado sobre la base conceptual anteriormente abordada. Se partió de la siguiente definición de competencias: "Competencia son aquellas habilidades integrales en el que se logran incorporar el saber ser (ética, valores), el saber hacer (habilidades, destrezas), el saber conocer (habilidades cognitivas) y el saber convivir (habilidades de interactuar con los demás)". El instrumento se les envió por vía correo electrónico.

Sobre la base de este concepto, las personas respondieron preguntas abiertas relacionadas con la percepción de las competencias deseables en el colectivo profesional de la Orientación y el criterio profesional de las competencias importantes de desarrollar en el colectivo de Orientación, con lo cual resultan los principales hallazgos de la consulta, expuestos en la Tabla 3. 
Según reflejan los resultados la Tabla 3 y Figura 1, sobre las competencias deseables en un profesional de la Orientación, un 58,00\% considera que la comunicación oral y escrita y asertiva es la principal competencia deseable en el profesional de la Orientación; un 42,00\% ubica las siguientes competencias: habilidades básicas de manejo del ordenador (tecnología), trabajo en equipo, liderazgo y confidencialidad. En un porcentaje menor, del 32,00\% se encuentran las habilidades interpersonales; con un $28,00 \%$, las competencias relacionadas con la empatía, conocimiento de un segundo idioma, iniciativa y aplicación de los conocimientos a la práctica, trabajo en equipo, creatividad y formación en ética, estética y ciudadanía; por último con un 26,00\%, la competencia de la responsabilidad y con un $24,00 \%$.la toma de decisiones.

Tabla 3

Principales hallazgos de la consulta

\begin{tabular}{|c|c|c|c|}
\hline $\begin{array}{l}\text { Competencias deseables en un } \\
\text { profesional de la orientación }\end{array}$ & Frecuencia & $\begin{array}{l}\text { Competencias que serían importantes } \\
\text { desarrollar en los y las orientados }\end{array}$ & Frecuencia \\
\hline Comunicación oral y escrita y asertiva & 29 & Trabajar en equipo & 14 \\
\hline $\begin{array}{l}\text { Habilidades básicas de manejo de } \\
\text { ordenador (tecnología) }\end{array}$ & 21 & Creatividad & 14 \\
\hline Trabajo en equipo & 21 & Responsabilidad & 13 \\
\hline Liderazgo & 21 & $\begin{array}{l}\text { Formación en ética, estética y } \\
\text { ciudadanía }\end{array}$ & 14 \\
\hline Confidencialidad & 21 & \multicolumn{2}{|l|}{ Observaciones: } \\
\hline Habilidades interpersonales & 16 & \multicolumn{2}{|l|}{ Frecuencia $29=58,00 \%$} \\
\hline Empatía & 14 & \multicolumn{2}{|l|}{ Frecuencia $21=42,00 \%$} \\
\hline Conocimiento de un segundo idioma & 14 & \multicolumn{2}{|l|}{ Frecuencia $16=32,00 \%$} \\
\hline Toma de decisiones & 12 & \multicolumn{2}{|l|}{ Frecuencia $14=28,00 \%$} \\
\hline Iniciativa & 14 & \multicolumn{2}{|l|}{ Frecuencia $13=26,00 \%$} \\
\hline Aplicar los conocimientos a la práctica & 14 & \multicolumn{2}{|l|}{ Frecuencia $12=24,00 \%$} \\
\hline
\end{tabular}

Nota. Elaboración propia sobre la base de los resultados de la consulta. 
Figura 1

Principales hallazgos de la consulta.

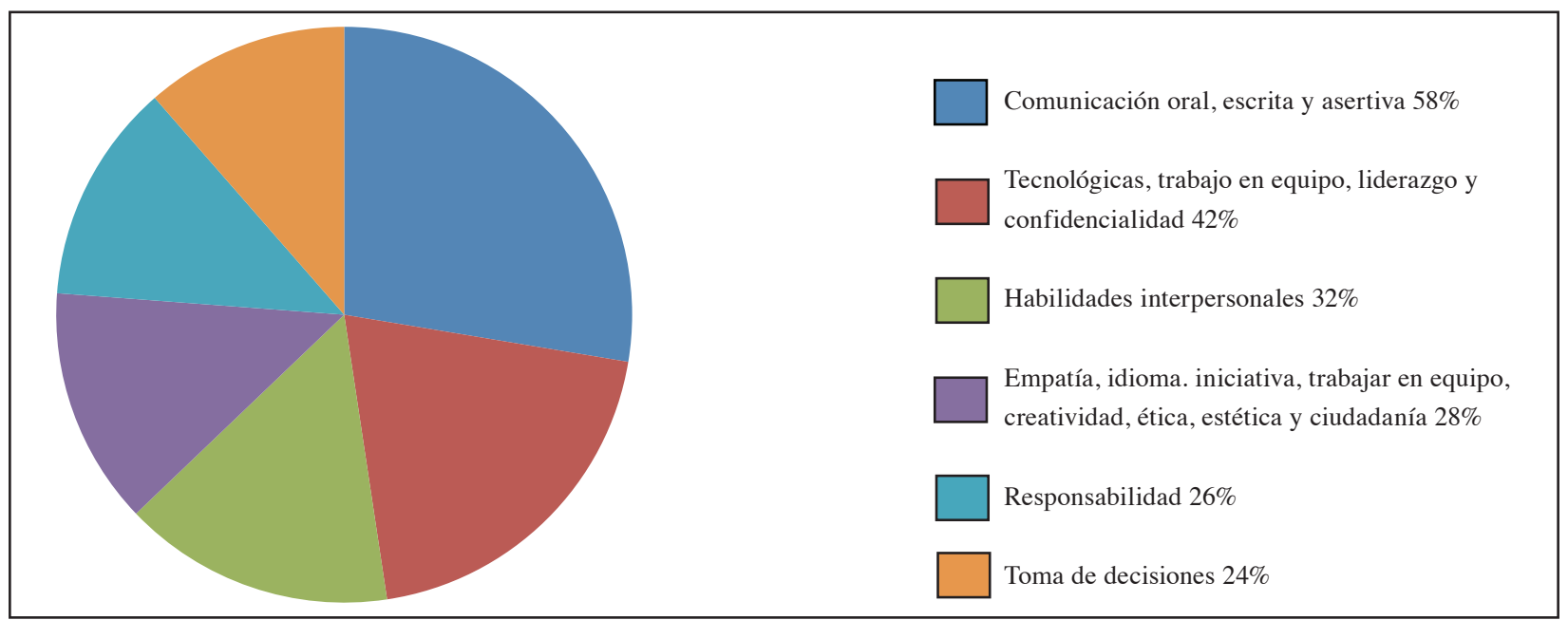

Nota. Elaboración propia sobre la base de los resultados de la consulta.

En relación con las competencias consideradas por los profesionales consultados, importantes de desarrollar en las personas orientadas, se establecen con mayor frecuencia el trabajo en equipo y, en segundo lugar, la creatividad, responsabilidad y la formación en ética, estética y ciudadanía.

En la Tabla 4, se establecen los principales hallazgos específicos que generó la consulta efectuada, tanto para las competencias deseables en el grupo profesional de la Orientación, como en las personas orientadas.

Tabla 4

Hallazgos específicos que generó la consulta efectuada

\begin{tabular}{|l|l|}
\hline Competencias deseables en un profesional de la Orientación & $\begin{array}{l}\text { Competencias que serían importantes desarrollar en las } \\
\text { personas orientadas }\end{array}$ \\
\hline Preocupación por la calidad & $\begin{array}{l}\text { Iniciativa } \\
\text { Toma de decisiones }\end{array}$ \\
Motivación & Integrar la teoría y la práctica \\
Compromiso ético & Trabajo manual \\
Habilidades de gestión de la información & Trabajo intelectual \\
Investigación & Capanomía \\
Aprender a aprender & Liderazgo continua \\
Análisis y síntesis, conocimientos básicos y específicos & Mantenerse actualizado en un mundo cambiante \\
Organización & Compañerismo \\
Capacidad crítica & Conocimiento de normativas y leyes \\
Conocimiento de leyes nacionales e internacionales relacionadas & Puntualidad \\
labor diaria & Conocimiento de derechos y deberes \\
Inteligencia emocional & Capacidad de escucha \\
Planificación y toma de decisiones & Comunicación asertiva \\
Compromiso con la institución donde se labora & Manejo de presión de grupo \\
Comunicativo, conciliador, sensibilidad, agudeza, creatividad. & Respeto \\
Responsabilidad & Humildad \\
Puntualidad & Manejo de recursos tecnológicos \\
Disponibilidad fuera de horario laboral & \\
Involucrarse con la realidad del estudiante y docentes & \\
Justo, equilibrado, decidido, respetuoso & \\
Afrontar retos & \\
Disposición al cambio & \\
Apertura a la diversidad & \\
\hline
\end{tabular}

Nota. Elaboración propia sobre la base de los resultados de la consulta. 


\section{Análisis de la consulta}

Las competencias que establecen los orientadores que realizaron la consulta refieren más a competencias genéricas que específicas, cabe rescatar que no se hace alusión a los procesos vocacionales como pilar de esta disciplina.

Existe una claridad en los profesionales de la Orientación consultados en que la principal competencia que se requiere es la comunicación. Todas las demás competencias expuestas presentan una congruencia entre lo que propone la Asociación Internacional para la Orientación Educativa y Profesional que se ha tenido como referencia en el presente estudio.

En relación con los individuos orientados, se hace alusión, en primer lugar, al trabajo en equipo y se comparten, con el profesional en Orientación, otras como liderazgo, creatividad, puntualidad y escucha.

La diversidad de criterios acerca de las competencias que consideran los orientadores y orientadoras es muy compleja, aun con una muestra tan pequeña no se evidencia en detalle las competencias específicas deseables tanto en la población profesional de esta disciplina como en la población dicente, las cuales sí son más claras y desarrolladas en la propuesta que ofrece la AIOEP.

Según los datos obtenidos, estas competencias de acercan más al análisis de las mismas en las categorías que ofrece Abdón (2003) desde las 4 dimensiones de ubicación de las competencias básicas. Específicamente, las competencias que se presentan en estos resultados se vinculan a las competencias genéricas en las dimensiones intelectual, dimensión social y dimensión interpersonal.

\section{Consideraciones finales}

El análisis del enfoque por competencias es incipiente en la disciplina de la Orientación. Se ha conocido a través de bibliografía con más profundidad un enfoque de habilidades, valores e intereses, creencias y actitudes, motivaciones, aptitudes y rasgos de personalidad.

El enfoque por competencias todavía no se encuentra aplicado en su profundidad en Costa Rica, por tanto, se puede hablar de un enfoque por competencias, donde solo algunos elementos del currículo tienen este elemento.

Los procesos realizados en Europa y en los últimos años en América Latina, tienen y tendrán una incidencia significativa en el sistema educativo costarricense, asimismo estarán ligados a las demandas de empleabilidad que se dicten desde los sistemas políticos, económicos y sociales. Por ende y desde la orientación vocacional, el protagonismo de la disciplina, una vez más, estará fundamentada en este pilar, en estos procesos de Bolonia y de Tuning de América Latina, donde sí se expresa una competitividad en el enfoque por competencias, le siguen los grandes intereses de las empresas y corporaciones mundiales, donde, sin lugar a dudas, se verá involucrado todo el sistema educativo costarricense. Asimismo no se concibe, por ninguna razón, la discriminación de ningún individuo en una sociedad donde la influencia neoliberal se ha visto impregnada en las políticas públicas para el desarrollo del país; cabe rescatar que en un país de derecho todos y todas las ciudadanas tienen un lugar importante donde se es útil por el solo hecho de existir.

Existe una vinculación entre el perfil ideal de competencias del profesional y el real desempeño laboral que ejercen los profesionales de la Orientación. Se debe partir del supuesto de criticidad ante el enfoque por competencias y no desde la aceptación sin análisis profundo de lo que se quiere; por una parte y desde la que parte el estudio: de una visión integral, humanista, donde todos y todas 
poseen un lugar; y el otro escenario, desde las demandas económicas, políticas y sociales que se enfrentan y enfrentarán en el siglo XXI.

No existe, hasta el momento, una categorización por parte del Servicio Civil de Costa Rica, encargado de los perfiles de contratación, que establezca un perfil de competencias para contratar profesionales en Orientación. Se resume en la Figura 2, que el orientador u orientadora del Siglo XXI requiere identificar tanto los retos y demandas del contexto, como los modelos, enfoque y teorías que fundamentan la disciplina de la Orientación, identificando los requerimientos y las características del campo laboral del Siglo XXI para dar respuesta a las competencias que este profesional y su orientados y orientadas requieren.

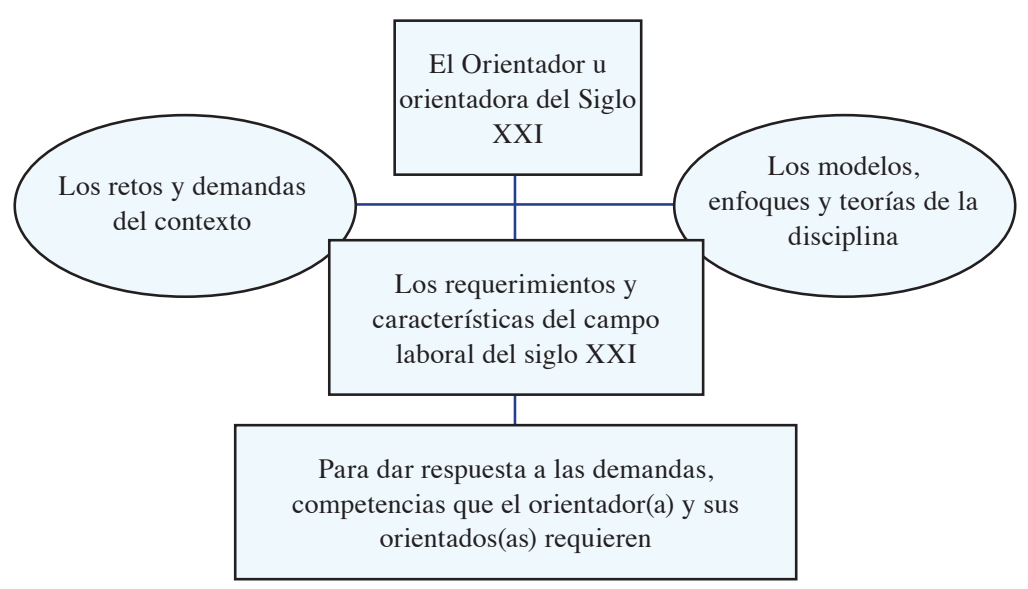

Figura 2

El o la profesional de la orientación en el siglo XXI.

Nota. Elaboración propia.

Las demandas actuales de los contextos dinámicos y complejos a los que se tienen que enfrentar tanto profesionales como orientados y orientadas, requieren del desarrollo de competencias para la vida, basadas en el respeto y la diversidad.

Todas las competencias son importantes; sin embargo, no todas están tan desarrolladas o no todas se pueden potencializar por el ambiente laboral, quiere decir que, por factores personales, contextuales o de formación, tienen un papel preponderante en el desarrollo de las mismas.

Los mecanismos de evaluación de las competencias profesionales en Costa Rica se limitan a las evaluaciones realizadas a finales de año por parte del principal empleador que es el Ministerio de Educación Pública. No se da una evaluación en proceso, ni de realimentación, sobre todo por el empleador del sector público. Con algunas diferencias del sector privado que realizan valoraciones de éxito en el proceso de laboral del profesional, sobre todo en su desempeño este profesional.

Se requiere de una aprobación de las competencias genéricas y específicas que se necesitan para el desempeño del profesional o la profesional de la Orientación por parte de los actores y las actoras en el campo laboral.

Desde el enfoque por competencias, se plantean una serie de retos a las universidades que forman a los orientadores y orientadoras, resumidos a continuación:

- Ofrecer un proceso de enseñanza-aprendizaje pertinente a las demandas de la actualidad.

- Involucrarse de lleno en el manejo de las nuevas tecnologías. 
- Desarrollar la comunicación asertiva en los procesos de aprendizaje, desde una vivencia de lo cotidiano.

- Enseñar el trabajo en equipo.

- Desarrollar las habilidades del liderazgo.

- Fortalecer la docencia con estrategias de enseñanza del trabajo inter-multi y transdisciplinar.

En conclusión, la eficacia del enfoque con miras a ser operacionalizado desde un modelo por competencias, no es solo una situación semántica sino de fondo, que requiere de un replanteamiento serio de los fines de la educación.

Las competencias relacionadas con el saber ser, son las que, a criterio de los autores, benefician integralmente al individuo, a los demás y a su contexto; el desarrollo de estas competencias trasciende el escenario personal llevando beneficios tangibles al contexto laboral.

El apartado de las competencias que, al parecer, no tienen valor científico por determinarse románticas, logra tener un sentido mayor y funcional en este estudio, dado que los espacios del ser en relación con otros son fundamentales para el devenir de nuevas formas de concebir la disciplina de la Orientación, de interactuar y de hacer las cosas. Solo el hecho de dedicar un tiempo a la escucha activa en un escenario informal con un colega o una colega, puede ser producto de una buena y útil idea, de una teoría, de un enfoque o de una intervención.

Empezar a validar y reforzar las propias competencias que tienen los orientadores y orientadoras en la actualidad es el comienzo de un aporte valioso del actual modelo por competencias que impera a nivel mundial.

\section{Referencias bibliográficas}

Abdón, I. (2003). Aprendizaje y Desarrollo de Competencias. Colombia: Cooperativa Editorial Magisterio.

Benitone, P., Esquetini, C., González, J., Maletá, M. M., Siufi, G., Wagenaar, R. [Eds.] (2007). Reflexiones y Perspectivas de la Educación Superior en América Latina. Informe Final -Proyecto Tuning-América Latina 2004-2007. España: Publicaciones de la Universidad de Deusto.

Delors, J. (1996). La educación encierra un tesoro. Informe a la UNESCO de la Comisión Internacional sobre la Educación para el Siglo XXI. París: Ediciones UNESCO. Recuperado de http://www.unesco.org/education/pdf/DELORS_S.PDF

Gardner, H. (1998). Inteligencias múltiples. La teoría en la práctica. Barcelona, Paidós.

González, J. y Wagenaar [Ed.]. (2008). Universities' contribution to the Bologna Process. An introduction: Universidad de Deusto (2a ed.). España: Universidad deDeusto. Recuperado de http:// tuning.unideusto.org/tuningeu/

Ministros Europeos de Educación. (1999, Junio, 19). Declaración conjunta de los Ministros Europeos de Educación reunidos en Bolonia. Bolonia. 
Ministros de Educación Superior. (2007). Comunicado de Londres. Hacia el Espacio Europeo de Educación Superior: respondiendo a los retos de un mundo globalizado. Recuperado de http://www.crue.org/export/sites/Crue/procbolonia/documentos/antecedentes/Comunicado_ de_Londres_2007.pdf

Ministros Europeos en Funciones de la Educación Superior. (2001). Declaración de Praga, 2001. Hacia el Área de la Educación Superior Europea. Recuperado el 1 de diciembre, 2010, en http://www.ond.vlaanderen.be/hogeronderwijs/bologna/links/language/2001 Prague Communique Spanish.pdf

Ministros Europeos de Educación Superior. (2003). Proceso de Bolonia. Realizando el Espacio Europeo de Educación Superior. Comunicado oficial de la Conferencia de Ministros responsables de Educación Superior, en Berlín. Berlín. Recuperado de http://www. google.co.cr/url? sa=t\&source $=$ web $\& \mathrm{~cd}=6 \&$ ved $=0 \mathrm{CDoQFjAF} \& u r l=$ http $\% 3 \mathrm{~A} \% 2 \mathrm{~F} \% 2 \mathrm{Fww}$ w.uco.es $\% 2$ Forganizacion $\% 2 \mathrm{Feees} \% 2 \mathrm{Fdocumentos} \% 2 \mathrm{Fnormas}$-documentos $\% 2 \mathrm{Fdoc}$-basi ca\%2FComunicado\%2520de\%2520Berlin\%25202003.pdf\&rct=j\&q=Ministros $\% 20 \mathrm{de} \% 20$ Educaci\%C3\%B3n\%20Superior\%2C\%202003\%2BBer1\%C3\%ADn\&ei=pr3-TJrkFY76sAOqdyvCw\&usg=AFQjCNEsXcK47X tCttSidBG8H0u PYsAg\&cad=rja

Ministros Europeos de Educación Superior. (2009). El Proceso de Bolonia 2020 . El Espacio Europeo de Educación Superior en la nueva década. Comunicado de la Conferencia de Ministros europeos responsables de educación superior, Lovaina. Recuperado el 1 de diciembre, 2010 de: http://www.crue.org/export/sites/Crue/procbolonia/documentos/antecedentes/Comunicado_Lovaina_Ministerio_es.pdf

Ministros representantes de Francia, Alemania, Italia y el Reino Unido. (1998). Declaración de la Sorbona. Recuperado de http://www.eees.ua.es/documentos/declaracion_sorbona.htm

Profesional de la Orientación Educativa y Profesional [AIOEP].(2008). Competencias Internacionales para los Profesionales de Orientación de Educación. Berna. Recuperado el 3 de abril de 2010, de http://www.iaevg.org/iaevg/nav.cfm?lang=4\&menu=1\&submenu=5

Ruiz, N C. (2002). Desarrollo de Potencialidades y Competencias. Colombia: Editorial PROLIBROS.

Tobón, S. (2006). Competencias en la Educación Superior. Colombia: Ecoe Ediciones.

Tobón, S. (2010). Memorias. Mejora e innovación de la docencia, la investigación y la extensión en la educación superior desde el modelo de las competencias. (Material de apoyo a conferencia de CONARE.). Centro de Información en Formación y Evaluación. San José, Costa Rica: s. e.

Universidad Católica de Temuco (2005). Competencias Genéricas UC Temuco: Para la Formación Integral de Ciudadanos Socialmente Responsables. Temuco, Chile: Autor. 